\title{
Correção cirúrgica de perfuração esofágica traumática por vídeo-toracoscopia utilizando patch de pleura parietal e músculo intercostal
}

\section{Surgical correction of traumatic perforation by video-toracoscopy using patch of parietal pleura and intercostal muscle}

\author{
Fernando Kennedy Pereira Chaves $^{1}(\mathbb{D})$, Bruno Roberto da Silva Ferreira ${ }^{1}(\mathbb{D})$, Heladio Feitosa de Castro Filho ${ }^{2}$ (D), Gotardo \\ Duarte Dumaresq ${ }^{2}$ (D) , Francisco Martins Neto ${ }^{2}$ (1) \\ 1. Médico Residente de Cirurgia Geral do Instituto Doutor José Frota (IJF), Fortaleza, CE, Brasil. 2. Médico Cirurgião do Instituto Doutor José Frota (IJF), \\ Fortaleza, CE, Brasil
}

\section{Resumo}

Introdução: As lesões de esôfago são consideradas graves. Avanços técnicos permitiram aplicar técnicas de cirurgia minimamente invasiva nesse tipo de lesão. Relato de caso: mulher, 22 anos, vítima de perfuração por projétil de arma de fogo transfixante. Após avaliação inicial, foi submetida à toracostomia à direita por hemopneumotórax. No segundo dia de internamento, após o início da dieta por via oral, foi flagrada uma saída de secreção mucoide pelo dreno. A paciente foi submetida à videotoracoscopia à direita, sendo realizada sutura da lesão esofágica associada a patch de pleura parietal e músculo intercostal. Conclusão: a abordagem por videotoracoscopia mostra-se segura e eficaz.

Palavras-chave: Doenças do Esôfago. Traumatismos Torácicos. Toracoscopia.

\begin{abstract}
Introduction: Esophageal injuries are considered serious. Technical advances allowed the application of minimally invasive surgery techniques in this type of lesion. Case report: Woman, 22 years old, victim of transfixing gunfire wound. After initial evaluation, patient was submitted to right thoracostomy due to hemopneumothorax. On the second day of hospitalization, after starting oral diet, the elimination of mucoid secretion from the chest drain was detected. Pacient underwent right-sided videothoracoscopy, in which suturing of an esophageal lesion was performed in association with a parietal pleura and intercostal muscle patch placement. Conclusion: Thoracoscopy approach is safe and effective.
\end{abstract}

Key words: Esophageal Diseases.Thoracic Injuries.Thoracoscopy.

\section{INTRODUÇÃO}

O trauma esofágico agudo é uma terrível condição cirúrgica com risco de morte, apresentando uma morbidade e mortalidade historicamente elevadas e é primordial para a detecção rápida da lesão ${ }^{1}$.

O tratamento cirúrgico da perfuração de esôfago é feito classicamente por toracotomia aberta. Nas últimas décadas, a videotoracoscopia começou a ser frequentemente utilizada em todo o mundo para diagnóstico e tratamento de afecções torácicas no trauma ${ }^{2,3}$.

Avanços técnicos permitiram aplicar técnicas de cirurgia minimamente invasiva que vêm mostrando, em vários casos, menor morbidade e mortalidade, menores incisões, menor tempo de internação e mesmo tempo da cirurgia da abordagem convencional ${ }^{1,3}$.

Dessa forma, este estudo teve como objetivo relatar o caso de um paciente que apresentou perfuração esofágica traumática por projétil de arma de fogo corrigida por videotoracoscopia.

\section{RELATO DO CASO}

Mulher, 22 anos, parda, apresentou trauma torácico penetrante por projétil de arma de fogo com entrada em hemitórax direito (HTD), e saída em hemitórax esquerdo (HTE) e ombro direito. Deu entrada no departamento de emergência apresentando dor torácica e dispneia. Ao exame inicial, taquipneico, taquicárdico e com murmúrio vesicular, diminuído em HTD, associada à percussão maciça. Após avaliação inicial, foi submetida à toracotomia fechada sob selo d'água à direita por hemopneumotórax e internada na enfermaria.

No segundo dia do pós-operatório, após início da dieta por via oral, foi flagrada uma saída de secreção mucoide pelo dreno, juntamente com restos alimentares, sendo aventada hipótese de lesão de trato digestivo. Paciente iniciou quadro de sepse. Foi realizada endoscopia digestiva alta que mostrou orifício de perfuração em esôfago distal, logo acima da transição esofagogástrica. Realizou-se uma tomografia de tórax, mostrando derrame pleural bilateral associado à atelectasia do parênquima adjacente (figura 1). 
Figura 1. Tomografia do tórax.

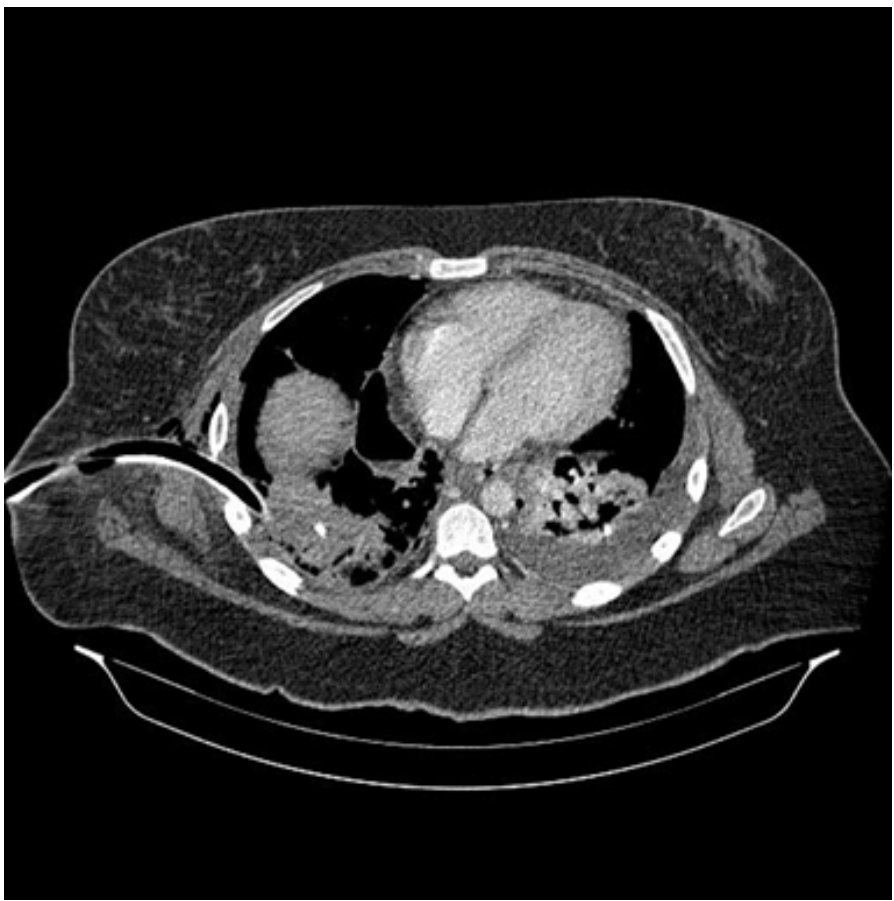

Após a avaliação do serviço de cirurgia torácica, foi indicado procedimento cirúrgico. A paciente foi submetida à videotoracoscopia à direita. O paciente foi posicionado em decúbito lateral esquerdo. Foram feitos quatro portais para a aposição dos trocateres, realizada decorticação pleural, abertura de mediastino posterior com saída de secreção purulenta e saliva (figura 2), liberação do esôfago com visualização direta da lesão com sutura da lesão esofágica com patch de pleura parietal e musculatura intercostal que funcionou como retalho (figura3).

Figura 2. Lesão do esôfago.

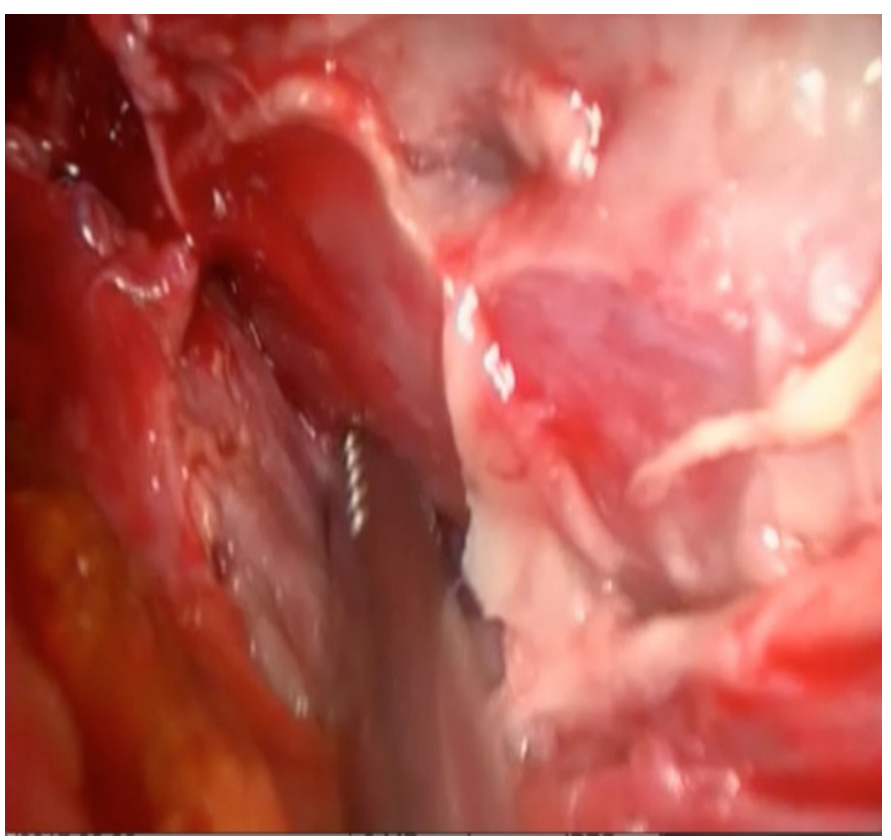

Figura 3. Patch de pleura parietal e musculatura intercostal.

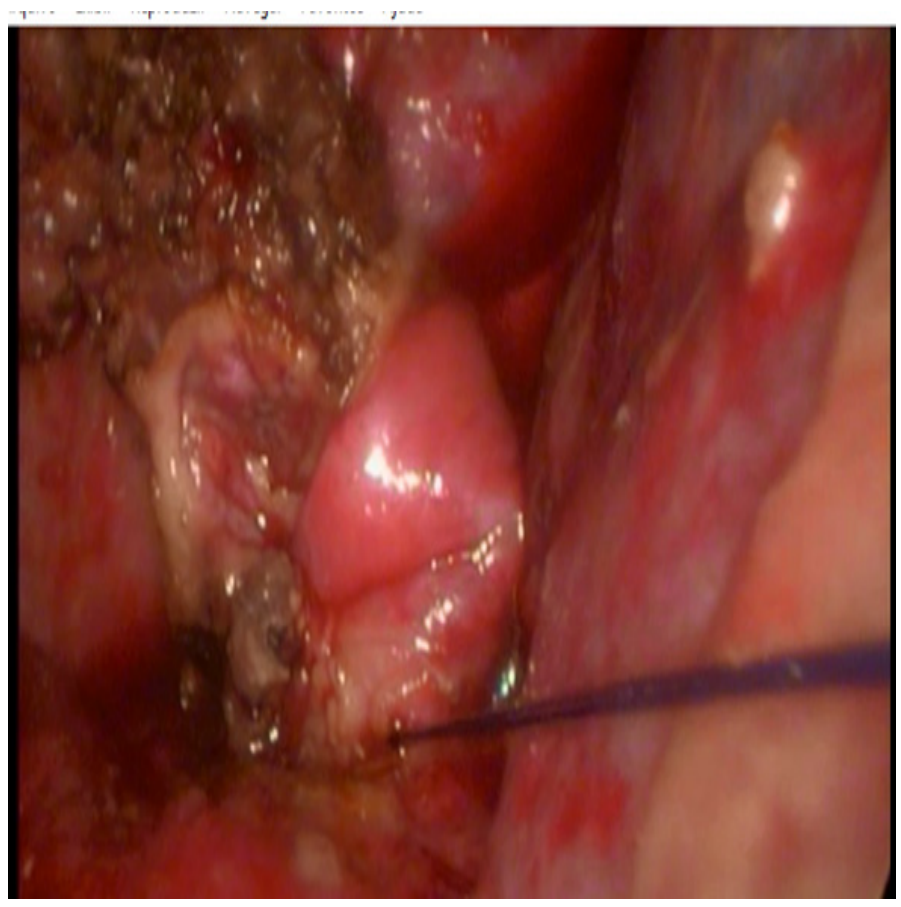

Terminada a sutura, realizou-se uma inspeção ampla da cavidade, com revisão hemostática rigorosa e posicionamento de dreno torácico. Foi deixada a paciente com sonda nasoenteral. Após dois dias da cirurgia inicial, foi necessário fazer uma decorticação pulmonar por videotoracoscopia à esquerda por empiema pleural, com limpeza de toda a cavidade pleural. A paciente evoluiu com uma ótima expansão pulmonar.

A paciente foi deixada em toracostomia fechada sob selo d'água bilateral, sendo alimentada por sonda nasoenteral, permanecendo em dieta por via oral zerada. Foi realizado teste com a ingestão de azul de metileno no sétimo pós-operatório, que foi negativo para fístula esofágica. Foi iniciada dieta por via oral com boa aceitação e sem saída da dieta pelo dreno. A paciente evoluiu bem no pós-operatório, tendo alta no décimo segundo dia do pós-operatório. No seguimento ambulatorial, encontra-se assintomática e com ganho de peso.

\section{DISCUSSÃO}

A abordagem da perfuração esofágica é complexa e ainda desperta muitas controvérsias. As causas de perfuração de esôfago são extremamente heterogêneas e há uma grande variação de abordagem de acordo com o tipo de lesão, tempo para abordagem inicial, tamanho e localização da ruptura, estado geral do paciente e experiência do cirurgião.

Independentemente da causa da perfuração do esôfago, a sua ruptura gera contaminação dos espaços circundantes por secreções gástricas e orais. Essa situação leva à inflamação local que pode evoluir para sepses e está associada à morbidade e à mortalidade significativas ${ }^{4,5}$. 
É importante salientar que, anualmente, há poucos casos de lesões de esôfago em instituições individuais (média de 3,9 casos por ano) e, portanto, o tratamento dessa lesão é limitado, muitas vezes, a grandes centros de traumas com cirurgiões treinados5. Assim, é importante destacar que o hospital no qual foi realizado o estudo possui cirurgiões experientes em abordagem desse tipo de trauma.

Tradicionalmente, a cirurgia de perfuração de esôfago é realizada por toracotomia. Esses pacientes apresentam alto risco de desenvolver complicações no pós-operatório. Vários estudos recentes estão sugerindo que a abordagem menos agressiva tem resultados com menor mortalidade, tempo de internação e qualidade de vida. Essas terapias são principalmente a sutura primária, cirurgia minimamente invasiva, stent endoscópico e até terapia conservadora em pacientes com perfuração esofágica ${ }^{3,5,6,7}$.

Os resultados cirúrgicos para o tratamento com videotoracoscopia são semelhantes aos da toracotomia convencional com a realização de sutura primária no esôfago em pacientes, por exemplo, com síndrome de Boerhaave 7,8. As técnicas toracoscópicas podem estar associadas a menores estresses cirúrgicos, menor tempo de permanência hospitalar, redução do trauma cirúrgico, menor dor pós-operatória, além de permitir drenagem ampla do mediastino ${ }^{3,5,7}$.

Uma análise retrospectiva com 16 pacientes com diagnóstico tardio de perfuração do esôfago mostrou que abordagem cirúrgica por videotoracoscopia é capaz de controlar a infecção pleural, a expansão pulmonar e possibilitar a completa regeneração do esôfago com perfuração diagnosticada tardiamente. Foram incluídos, neste estudo, todos os pacientes com diagnóstico tardio ( $>12$ horas) de perfuração do esôfago torácico tra-tados por videotoracoscopia, sem sutura ou ressecção do órgão, em um período de 15 anos $^{11}$. Entretanto, há relatos mostrando aumento acentuado da mortalidade $(20,0 \%)$ em pacientes submetidos a qualquer outro reparo, quando comparado com o reparo primário com uma taxa de mortalidade de $9,5^{5}$.

O paciente do caso já possuía infecção pleural e sepse grave, porém foi decidida a realização de sutura esofágica associada a patch de pleura parietal e músculo intercostal, que funcionou como um retalho, já que patch com estrutura muscular tem uma melhor cicatrização pela vascularização mantida.

Já no câncer de esôfago, um estudo randomizado com Followup de 3 anos mostrou que a cirurgia minimamente invasiva tem menos complicações pulmonares e uma melhor qualidade de vida na pós-operatória no acompanhamento de curto prazo. Já, no longo prazo, mostrou um resultado igualmente seguro em relação à sobrevida e à recidiva da doença ${ }^{9}$.

A educação sólida e um bom conhecimento da videocirurgia e trauma torácico são pré-requisitos essenciais para a prática dessas técnicas em feridas do tórax ${ }^{10}$. Além do que, o nó intracorpóreo e extracorpóreo requer habilidades toracoscópicas avançadas ${ }^{3}$. Neste relato, o procedimento foi realizado por um cirurgião torácico com experiência em cirurgia minimamente invasiva de tórax.

Em relação ao prognóstico, uma análise comparativa de uma metanálise mostrou que o atraso maior que 24 horas no diagnóstico e a abordagem das lesões esofágicas estão associados a uma risk ratio de $2,28^{5}$. Dessa forma, o tempo entre a perfuração e o diagnóstico é considerado o fator mais importante no tratamento das lesões do esôfago ${ }^{4,5,6}$. O paciente do caso, apesar de ter sido abordado depois de 24 horas, ainda houve condições técnicas para realizar a sutura primária. Assim, foi possível evitar a esofagectomia e confecção de tubo gástrico em um segundo tempo que tem uma maior mortalidade nessa abordagem quando comparada à sutura primária 5 .

A paciente deste relato já estava em sepses, com pulmões comprometidos e coletando secreções, apesar de o tempo não ser o ideal para a abordagem, ainda foi possível a intervenção e sutura com patch de pleura parietal e musculatura intercostal.

Existe ainda a possibilidade de colocação de stent endoscópico. Relatórios sobre bons resultados, usando stents de grande diâmetro em lesões de perfurações esofágicas traumáticas não malignas de esôfago concluem que podem ser usados com segurança nesta população, apesar de não ter mostrado a superioridade dessa abordagem sobre os outros tratamentos 5 . Em nossa realidade, além da dificuldade para adquirir o stent em tempo hábil, a sua colocação seria dificultada pela lesão ser distal próximo à cárdia, assim, não sendo utilizado nesse paciente.

Assim, o tratamento das lesões complexas de esôfago é desafiador para o cirurgião. Nessa paciente, a lesão traumática de esôfago tratada tardiamente por vídeo-laparoscopia obteve um ótimo resultado logo após a cirurgia, com controle da sepse, fechamento da fistula esofagiana e expansão pulmonar. Houve, assim, uma melhora relativamente rápida da paciente.

A videotoracoscopia pode evitar a toracotomia em expressivo número de pacientes submetidos ao procedimento em mãos de cirurgiões habilitados para essa técnica. O tempo entre a ruptura e o tratamento pode ter importantes implicações terapêuticas e prognósticas.

Contudo, estudos adicionais são necessários com dados mais detalhados para identificar fatores de risco associados a resultados ruins e avaliar melhor a eficácia das atuais modalidades de tratamento, principalmente, na abordagem por videotoracoscopia nos pacientes diagnosticados tardiamente. 


\section{REFERÊNCIAS}

1. Lang-Lazdunski L, Mouroux J, Pons F, Grosdidier G, Martinod E., Elkaim D, Azorin J., Jancovici R. Role of videothoracoscopy in chest trauma. Ann Thora Surg. 1997; 63(2):327-333.

2- Tjardes T, Wafaizadeh A, Steinhausen E, Krakamp B, Bouillon B. Extension injury of the thoracic spine with rupture of the oesophagus and successful conservative therapy of concomitant mediastinitis. Eur Spine J 2009 Jul; 18 (Suppl 2):240-244. doi: 10.1007/s00586-009-0930-z.

3- Nakano T, Sato C, Sakurai T, Kamiya K, Kamei T, Ohuchi, N. Thoracoscopic esophageal repair with barbed suture material in a case of Boerhaave's syndrome. J Thorac Dis. 2016 Dec; 8(12): E1576-E1580. doi: 10.21037/ jtd.2016.12.46.

4- Marsico GA, Azevedo DED, Guimarães CA, Mathias I, Azevedo LG, Machado T. Injuries of the esophagus. Rev Col. Bras. Cir. 2003; 30(3):216-223.

5-Biancari F, D'Andrea V, Paone R, et al. Current Treatment and Outcome of Esophageal Perforations in Adults: Systematic Review and Meta-Analysis of 75 Studies. World J Surg. 2013 May; 37(5):1051-1059. doi: 10.1007/s00268-0131951-7.

6-Ben-David K, Behrns K, Hochwald S, Rossidis G, Caban A, Crippen C et al. Esophageal Perforation Management Using a Multidisciplinary Minimally
Invasive Treatment Algorithm. J Am Coll Surg. 2014 Apr; 218(4):768-774. doi: 10.1016/j.jamcollsurg.2013.12.033.

7- Cho JS, Kim YD, Kim JW, I HS, Kim MS. Thoracoscopic primary esophageal repair in patients with Boerhaave's syndrome. The Annals of thoracic surgery. 2011 May; 91(5):1552-1555. doi: 10.1016/j.athoracsur.2011.01.082.

8. Haveman JW, Nieuwenhuijs VB, Kobold JP, Van Dam GM, Plukker JT, Hofker HS. Adequate debridement and drainage of the mediastinum using open thoracotomy or video-assisted thoracoscopic surgery for Boerhaave's syndrome. Surg Endosc 2011 Aug; 25(8): 2492-2497. doi: 10.1007/s00464-011-1571-y

9- Straatman J, van der Wielen N, Cuesta MA, Daams F, Garcia JR, Bonavina L, et al. Minimally invasive versus open esophageal resection: three-year follow-up of the previously reported randomized controlled trial: the TIME trial. Ann Surg. 2017 Aug; 266(2): 232-236. doi: 10.1097/SLA.0000000000002171.

10- Lang-Lazdunski L, Chapuis O, Pons F, Jancovici R; La vidéothoracoscopie dans les traumatismes et plaies du thorax. In: Annales de chirurgie. Elsevier Masson. 2003 Mar ;128(2): 75-80. doi: 10.1016 / S0003-3944 (02) 00039-1

11- Abou-Mourad OM, Andrade FMD, Júdice LF, Júdice Â, Carvalho ABCB Filho, Morard, MRS, et al. Video-thoracoscopic approach, without suture, of late thoracic esophageal perforations. Rev Col. Bras. Cir. 2017; 44(4);354-359.

\section{Como citar este artigo/How to cite this article:}

Chaves FKP, Ferreira BRS, Castro HFC Filho, Dumaresq GD, Martins F Neto. Correção cirúrgica de perfuração esofágica traumática por vídeo-toracoscopia utilizando patch de pleura parietal e músculo intercostal. J Health Biol Sci. 2018 Out-Dez; 6(4):463-466. 